\title{
Anti-Slug Control Experiments Using Nonlinear Observers*
}

\author{
E. Jahanshahi ${ }^{1}$, S. Skogestad ${ }^{1}$, E. I. Grøtli ${ }^{2}$
}

\begin{abstract}
To prevent slug-flow on offshore oil production units, controlling a subsea pressure is the recommended solution. However, the subsea pressure is not often available as a measurement. The top-side pressure is usually measured but it is difficult to use for stabilizing control, because of its Right Half-Plane zero dynamics combined with nonlinearity. We have used the top-side pressure as measurement for different observers to estimate state variables of the system, and then used the estimated states for control. This scheme was tested in experiments using three types of observers. A simple Luenberger observer with a large gain was found to be more robust than the standard Unscented Kalman Filter (UKF). We modified the UKF to a Fast UKF for time-scale separation of the closed-loop system. The resulting observer was robust, also less sensitive to measurement noise compared to the simple Luenberger observer when measuring the top-side pressure. Surprisingly, the nonlinear observers were not able to work in closed-loop when the subsea pressure was used as the measurement. On the other hand, a linear observer worked very well for this case with a larger operating range compared to that of the top-side pressure.
\end{abstract}

\section{INTRODUCTION}

The oscillatory flow condition in offshore multi-phase pipelines is undesirable and an effective solution is needed to suppress it [1]. Active control of the topside choke valve is a recommended solution to maintain a non-oscillatory flow regime together with the maximum possible production rate [2], and the control system used for this purpose is called anti-slug control. This control system uses measurements such as pressure, flow rate or fluid density as the controlled variables and the topside choke valve is the main manipulated variable.

It has been shown that it is difficult to stabilize flow in pipeline-riser systems when using only the top-side pressure measurement [3]. The reason is that the Right Half-Plane (RHP) zeros of the system with this measurement are relatively close to the RHP-poles of the system, and consequently the sensitivity transfer function of the system shows a large peak. On the other hand, the pressure measurements at the subsea are suitable control variables, and a simple PI controller is used in practice.

If only the top-side pressure measurement is available, the conventional control solution is to design an observer which uses the top-side pressure as the measurement and estimates the bottom pressure or states of the system, then

\footnotetext{
*This work was supported by SIEMENS, Oil \& Gas Division

${ }^{1}$ E. Jahanshahi, S. Skogestad are with Department of Chemical Engineering, Norwegian Univ. of Science and technology, Trondheim, NO-7491 skoge@ntnu. no

${ }^{2}$ E. I. Grøtli is with Department of Engineering Cybernetics, Norwegian Univ. of Science and technology, Trondheim, NO-7491 grotli@itk.ntnu.no
}

to use these estimates for control. However, the fundamental controllability limitation of the top-side pressure can not be bypassed completely by the observer. We want to see if this solution is applicable for anti-slug control, and which kind of observer is suitable for this purpose. In particular, we are interested to find out under which conditions and how observers can help to stabilize an unstable system with RHPzero dynamics in practice.

The Extended Kalman Filter has been used for estimating pressure at bottom of the well and state variables in gaslifted oil wells [4]. Moreover, a nonlinear observer based on back-stepping design was proposed for this purpose [5], and it was tested in experiments [6].

A nonlinear Luenberger-type observer which uses only topside pressure to reproduce oscillations of the riserslugging instability was proposed in [7], and then used for anti-slug control of a pipeline-riser system in experiments [8]. This observer was designed based on a three-state simplified model of the system [9]. It was found that the peak of the sensitivity transfer function of the topside pressure for their system was not large, and it did not impose limitations on the controllability [8]. However, this is true only for small valve openings, and we can not open the valve for large production rates when using the top-side pressure. The controllability of the top-side pressure is briefly investigated in this paper.

We use a four-state simplified model [10] for the observer and control design in this work. The model is fitted to experimental data by adjusting four parameters, and it shows good agreement with experiments.

Three types of observer were tested online on experiments using the top-side pressure. We used a Luenberger-type nonlinear high-gain observer, a standard Unscented Kalman Filter (UKF), and a UKF modified to incorporate the highgain observer concept [11] which we call Fast UKF.

The term "High-Gain observer" can cause confusion in some cases. One may say a observer is not high-gain, because the observer gain is $K=1$ [7]. However, it depends on which unit is used for the measurement; for example there is a scaling factor of $10^{3}$ between $\mathrm{Pa}$ and $\mathrm{kPa}$ for pressure.

This paper is organized as follows: The experimental rig and the simplified model for sever-slugging are presented in Section II. The three different observers used in this work are introduced in Section III. Experimental results are provided in Section IV and discussed in Section V. Finally, the main conclusions and remarks are summarized in Section VI. 


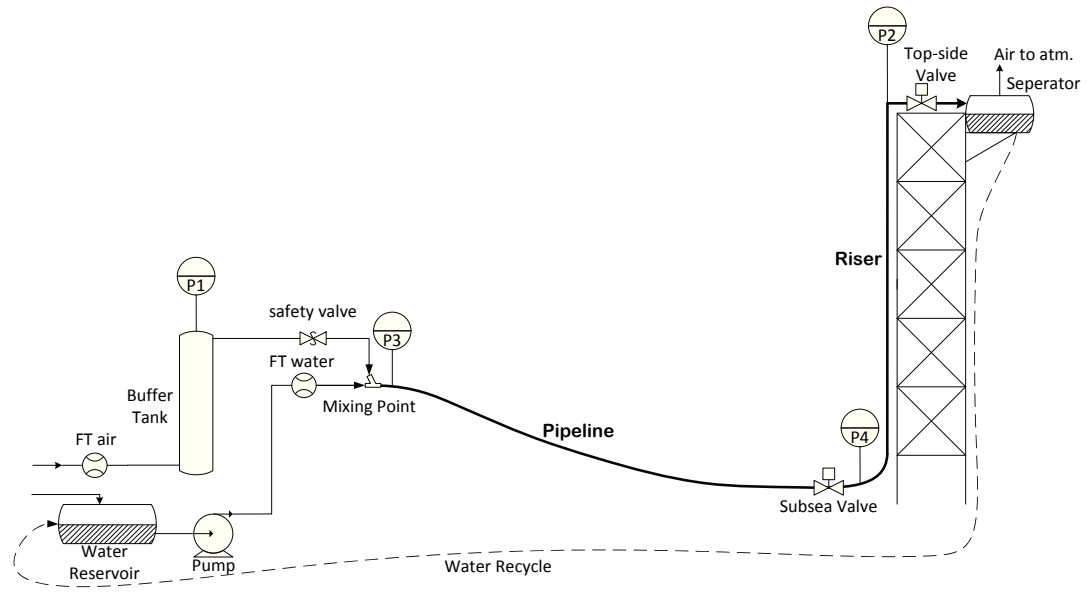

Fig. 1. Schematic diagram of experimental setup

\section{PIPELINE-RISER SYSTEM}

\section{A. Experimental setup}

The experiments were performed on a laboratory setup for anti-slug control at the Chemical Engineering Department of NTNU. Fig. 1 shows a schematic presentation of the laboratory setup. The pipeline and the riser in the L-shaped setup are made from flexible pipes with $2 \mathrm{~cm}$ inner diameter. The length of the pipeline is $5 \mathrm{~m}$, inclined with a $20^{\circ}$ angle, and height of the riser is $3 \mathrm{~m}$. A buffer tank is used to simulate gas expansion effect of a very long pipe with the same volume, such that the total resulting length of pipe would be about $70 \mathrm{~m}$.

The feed into the pipeline is considered at constant flow rates, 4 litre $/ \mathrm{min}$ of water and 4.5 litre $/ \mathrm{min}$ of air. With this boundary conditions, the system switches from stable (non-slugging) to unstable (slugging) operation at $15 \%$ opening of the top-side valve. The topside choke valve opening is used as the only control input, while the subsea valve is fully open in this work. The separator pressure after the topside choke valve is nominally constant at atmospheric pressure. The air is separated and goes to the atmosphere, and water is recycled back to the experiment loop.

\section{B. Simplified model}

A four-state simplified model for severe-slugging flow in pipeline-riser systems [10] is used for observer design and control. The state variables are

- $m_{g p}$ : mass of gas in pipeline

- $m_{l p}$ : mass of liquid in pipeline

- $m_{g r}$ : mass of gas in riser

- $m_{l r}$ : mass of liquid in riser

The state equations are the mass conservation laws:

$$
\begin{aligned}
\dot{m}_{g p} & =w_{g, \text { in }}-w_{g r, b} \\
\dot{m}_{l p} & =w_{l, \text { in }}-w_{l r, b} \\
\dot{m}_{g r} & =w_{g r, b}-w_{g, \text { out }} \\
\dot{m}_{l r} & =w_{l r, b}-w_{l, \text { out }}
\end{aligned}
$$
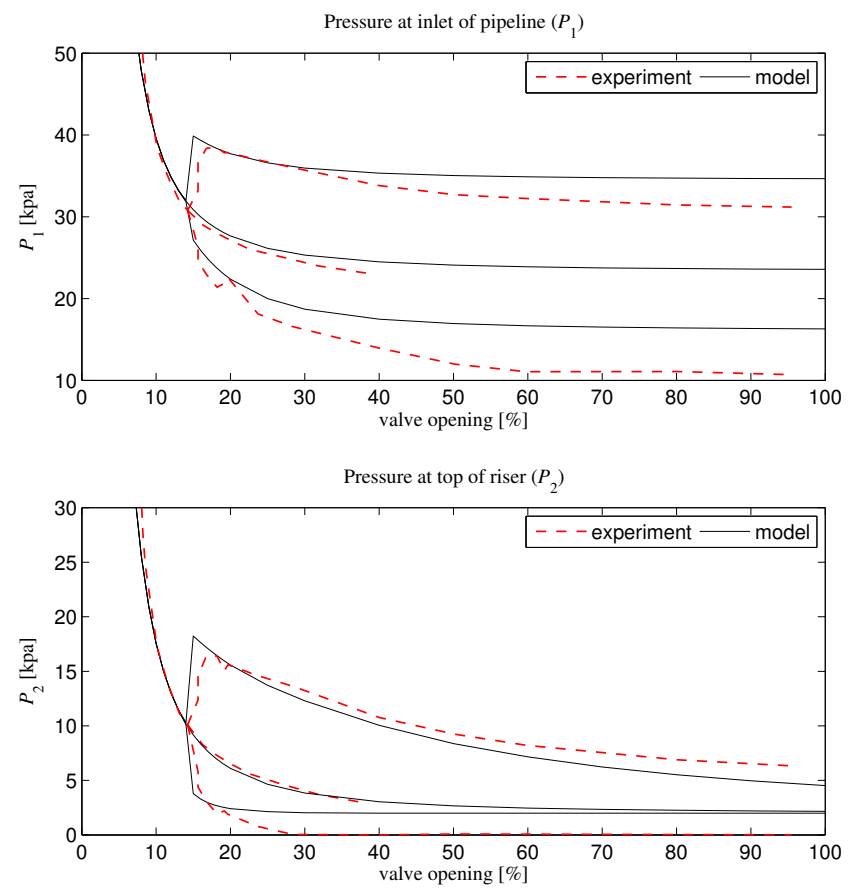

Fig. 2. Bifurcation diagrams of simplified model (solid) compared to experiments (dashed)

The flow rates in equations (1)-(4) are calculated by additional model equations which can be found in [10]. The simplified model was fitted to the experiments by adjusting the four parameters in the model:

- $K_{h}$ : correction factor for level of liquid in pipeline

- $K_{p c}$ : choke valve constant

- $K_{G}$ : coefficient for gas flow through low point

- $K_{L}$ : coefficient for liquid flow through low point

Bifurcations diagrams, describing the steady-state and the dynamics of this system, are used to compare the model to experiments [3]. Fig. 2 shows the bifurcation diagrams of the simplified model (solid lines) compared to the those of the 


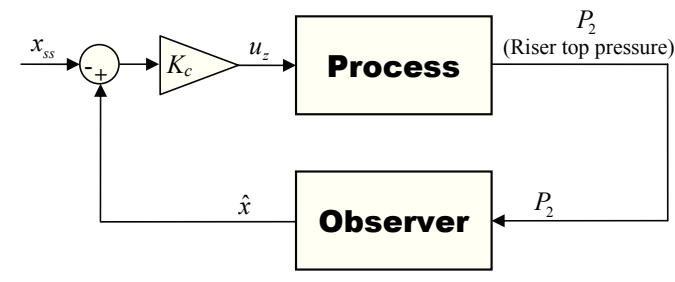

Fig. 3. Block diagram of closed-loop system

experiments (dashed lines). The system has a stable (nonslug) flow when the valve opening $Z$ is smaller than $15 \%$, and it switches to slugging flow conditions for larger valve openings. The minimum and maximum of the oscillations of the slugging together with the steady-state (in the middle) are shown in Fig. 2. The steady-state in slugging condition $(Z>15 \%)$ is unstable, but it can be stabilized by using control.

\section{OBSERVER DESIGN}

As illustrated in Fig. 3, we estimate the state variables of the system by using an observer. Then, we use the estimated states in a state-feedback controller. The separation principle allows us to separate the design into two tasks. First, we design a state-feedback controller that stabilizes the system and meets other design specifications. Then an output feedback controller is obtained by replacing the state $x$ by its estimate $\hat{x}$ provided by observers [11]. However, the separation principle does not hold in general for nonlinear systems. We examine the stability of closed-loop controler/observer by experiments. Further, we will investigate why and under which conditions the separation principle holds for the system under study. In the following, we present three observers used in experiments.

\section{A. Unscented Kalman Filter}

First, we consider the standard form of the Unscented Kalman Filter (UKF) as explained by [12]. The nonlinear state space system is given as

$$
\begin{gathered}
x_{k}=f\left(x_{k-1}, u_{k-1}\right)+v_{k-1}, \\
y_{k}=h\left(x_{k}, u_{k}\right)+w_{k},
\end{gathered}
$$

where $v_{k-1}$ is a vector of Gaussian zero-mean process noise, representing model error and disturbances, with the covariance matrix $Q_{k-1} . w_{k}$ is a vector of Gaussian zeromean measurement noise with the covariance matrix $R_{k}$.

We assume the state vector as a random variable with the mean value of $\hat{x}_{k-1}$ and the covariance matrix $P_{k-1}$, undergoing the nonlinear transform of $f$. The general problem is to find the statistics of this random variable after the nonlinear transform $\left(\hat{x}_{k}\right)$. The main idea behind the UKF, unlike the Monte Carlo simulations which need a large number of samples, is that the statistics of a nonlinear transformation can be found with fair accuracy by only a limited number of samples called sigma points. Samples in Monte Carlo simulations are chosen randomly, whereas in the UKF algorithm sigma points are chosen deterministically. Similar to the EKF, the UKF algorithm has a predictor/corrector nature, but we do not need to linearize the model by calculating the Jacobian matrices. The UKF algorithm is summarized in the following steps:

(1) Prediction step:

$\hat{X}_{k-1}=\left[\begin{array}{lll}\hat{x}_{k-1} & \ldots & \hat{x}_{k-1}\end{array}\right]+\sqrt{c}\left[\begin{array}{ll}\sqrt{P_{k-1}} & -\sqrt{P_{k-1}}\end{array}\right]$

$\hat{X}_{k}=f\left(\hat{X}_{k-1}, u_{k-1}\right)$

$\hat{x}_{k}^{-}=\hat{X}_{k} W_{m}$

$P_{k}^{-}=\hat{X}_{k} W_{c}\left[\hat{X}_{k}\right]^{T}+Q_{k-1}$

(2) Update step:

$\hat{X}_{k}^{-}=\left[\begin{array}{lll}\hat{x}_{k}^{-} & \ldots & \hat{x}_{k}^{-}\end{array}\right]+\sqrt{c}\left[\begin{array}{ll}\sqrt{P_{k}^{-}} & -\sqrt{P_{k}^{-}}\end{array}\right]$

$Y_{k}^{-}=h\left(\hat{X}_{k}^{-}\right)$

$\mu_{k}=Y_{k}^{-} W_{m}$

$S_{k}=Y_{k}^{-} W_{m}\left[Y_{k}^{-}\right]_{T}^{T}+R_{k}$

$C_{k}=\hat{X}_{k}^{-} W_{c}\left[Y_{k}^{-}\right]^{T}$

(3) Compute filter gain $K_{k}$ and updated state mean $\hat{x}_{k}$ and covariance $P_{k}$ :

$K_{k}=C_{k} S_{k}^{-1}$

$\hat{x}_{k}=\hat{x}_{k}^{-}+K_{k}\left[y_{k}-\mu_{k}\right]$

$P_{k}=P_{k}^{-}-K_{k} S_{k} K_{k}^{T}$

We do not discretize the model or the observers, instead we use ode15s solver in Matlab to integrate the model form time $k-1$ to $k$. Because of small dimensions of the laboratory set-up, the frequency of the unstable dynamics are relatively high, and we need a small sampling time $\left(T_{s}=0.1 \mathrm{sec}\right)$. With the four-state model, we get eight sigma points, and integrating the model eight times needs more computation time than the sampling time. We solved this problem by using parallel processing in Matlab.

\section{B. High-Gain Luenberger observer}

A high-gain observer, under certain conditions, guarantees that the output feedback controller recovers the performance of the state-feedback controller when the observer gain is sufficiently high. The observer gain is designed so that the observer is robust to uncertainties in modeling the nonlinear functions. The structure of the high-gain observer is similar to the one used in [7]:

$$
\begin{aligned}
& \dot{\hat{z}}_{1}=f_{1}(\hat{z}) \\
& \dot{\hat{z}}_{2}=f_{2}(\hat{z}) \\
& \dot{\hat{z}}_{3}=f_{3}(\hat{z})+\frac{1}{\epsilon}(y-\hat{y}) \\
& \dot{\hat{z}}_{4}=f_{4}(\hat{z})
\end{aligned}
$$

where

- $z_{1}$, mass of gas in pipeline $\left(m_{g p}\right)$

- $z_{2}$, mass of liquid in pipeline $\left(m_{l p}\right)$

- $z_{3}$, pressure at top of riser $\left(P_{2}\right)$

- $z_{4}$, mass of liquid in riser $\left(m_{l r}\right)$

and $\frac{1}{\epsilon}$ is the high-gain. Three of the equations $\left(f_{1}, f_{2}\right.$ and $f_{4}$ ) are same as the model in the (1), (2) and (4). For the third state equation $\left(f_{3}\right)$, we transformed the state into top pressure which is a measurement $\left(y=z_{3}\right.$ and $\left.\hat{y}=\hat{z}_{3}\right)$. We 
used $z_{3}=P_{2}$, because it is directly related to the third state of the model $m_{g r}$ (mass of gas in the riser):

$$
P_{2}=\frac{m_{g r} R T_{r}}{M_{G}\left(V_{r}-\frac{m_{l g}}{\rho_{l}}\right)}
$$

We only need to derive the time derivative of the top-pressure by using partial derivatives:

$$
\begin{gathered}
f_{3}(z)=\frac{d P_{2}}{d t} \\
\frac{d P_{2}}{d t}=\frac{\partial P_{2}}{\partial m_{g r}} \dot{m}_{g r}+\frac{\partial P_{2}}{\partial m_{l r}} \dot{m}_{l r}
\end{gathered}
$$

where

$$
\begin{gathered}
\frac{\partial P_{2}}{\partial m_{g r}}=\frac{a}{b-m_{l r}} \\
\frac{\partial P_{2}}{\partial m_{l r}}=\frac{a m_{g r}}{\left(b-m_{l r}\right)^{2}}
\end{gathered}
$$

In (10) and (11), $a=R T_{r} \rho_{l} / M_{G}$ and $b=\rho_{l} V_{r}$ are model constants. See [10] for details of the model.

\section{Fast UKF}

One advantage of the UKF over the simple high-gain observer is that the UKF relates the measurements to all the state equations with appropriate gains. Moreover, the Kalman Filter averages out measurement noise, and thus the estimates are less sensitive to noise. However, the standard UKF was not robust enough in closed-loop for our application. In most of the experiment runs the closed-loop system using the UKF was not able to stabilize the flow. However, by increasing the observer gain promising results were achieved. Although the observer gain for the UKF can be increased by using a small value for $R_{k}$ and a large value for $Q_{k-1}$, there is a limitation with this approach. By looking at the UKF algorithm, the observer gain is

$$
K_{k}=\frac{\hat{X}_{k}^{-} W_{c}\left[Y_{k}^{-}\right]^{T}}{Y_{k}^{-} W_{m}\left[Y_{k}^{-}\right]^{T}+R_{k}} .
$$

One can notice that the observer gain is highly dependent on the scale of states and measurements. For example, if measurements and states have the same magnitude or in the case of measuring just one of states, the maximum gain that the UKF can produce is 1. Even though the UKF, similar to the linear Kalman Filter, is optimal for estimation error in steady-state, it can not guarantee robustness of the closedloop system.

We aim to combine the advantages of the UKF with the robustness property of the high-gain observer. We implement this idea in a basic and simple way. We use the transformed model, similar to (6), instead of the original model in (1)(4). It is similar to the high-gain observer, but without the observer term.

$$
\begin{aligned}
& \dot{z}_{1}=f_{1}(z) \\
& \dot{z}_{2}=f_{2}(z) \\
& \dot{z}_{3}=f_{3}(z) \\
& \dot{z}_{4}=f_{4}(z)
\end{aligned}
$$

These are the model equations, only the third state has been transformed. We do not specify the observer term in state equations explicitly, neither do we determine the observer gain directly; we let the Unscented Transformation works its magic. The matrix $Q_{k}$ in the UKF algorithm represents process noises, and larger $Q_{k}$ leads to a large observer gain. The third state in the model shown by equation (13) is measured. By choosing suitable values for the elements of $Q_{k}$, we can put more weight in direction of the measured state. We construct $Q_{k}$ as follows:

$$
Q_{k}=\operatorname{diag}\left(q_{\min }, q_{\min }, q_{\max }, q_{\min }\right)
$$

where $q_{\min }$ and $q_{\max }$ are treated as tuning parameters. The UKF algorithm calculates the observer gains in an adaptive manner, and unlike the simple high-gain observer, it gives four elements corresponding to the four states. As suggested in [13], we incorporated the innovation information (Mean Square Error in a moving window) to make $R_{k}$ and $Q_{k}$ also adaptive. This idea was tested in simulations, by using OLGA simulator as the real process, also in experiments. We found that the closed-loop system is more robust in experiments by keeping $R_{k}$ and $Q_{k}$ constant.

Since we are using the UKF algorithm with a measured state $\left(y=z_{3}\right)$, the maximum observer gain is 1 . To have a better control on the observer gain, we scale the states and measurements of the system based on their steadystate values. All the states are normalized to 100 , and the measurement is normalized to 1000 . This scaling implies that when the UKF gives a gain of 1 , we have enforced a factor of 10. However, we show the measurements with their original scales in the experimental results.

\section{EXPERIMENTAL RESULTS}

We apply full state-feedback by using estimated states for control. In addition, to prevent drift from the operating point, an integral action is added by using the estimated subsea pressure. The total control action can be expressed as

$$
u(t)=-K_{c}\left(\hat{x}(t)-x_{s s}\right)+K_{i} \int_{0}^{t}\left(\hat{P}_{1}(\tau)-r\right) d \tau .
$$

We transform back the states from $\hat{z}$ to $\hat{x}$, then we use them for control. $K_{c}$ is a linear optimal controller calculated by solving Riccati equation and $K_{i}$ is a small integral gain (e.g. $K_{i}=10^{-3}$ ). The sampling time for the control is same as the sampling time of the observer $\left(T_{s}=0.1 \mathrm{sec}\right)$.

\section{A. Measuring top-side pressure}

The experimental result using the simple High-Gain Luenberger observer is shown in Fig. 4. The same experiment using the Fast UKF is shown in Fig. 5. Comparing Fig. 4 and Fig. 5, the Fast UKF has a better behavior and, unlike the high-gain observer, does not show any oscillation when the controller is on. The standard UKF was not able to stabilize the system for most of experiment runs, while the two other observers were successful in all experiment runs. The result of using the standard UKF is similar to Fig. 5 

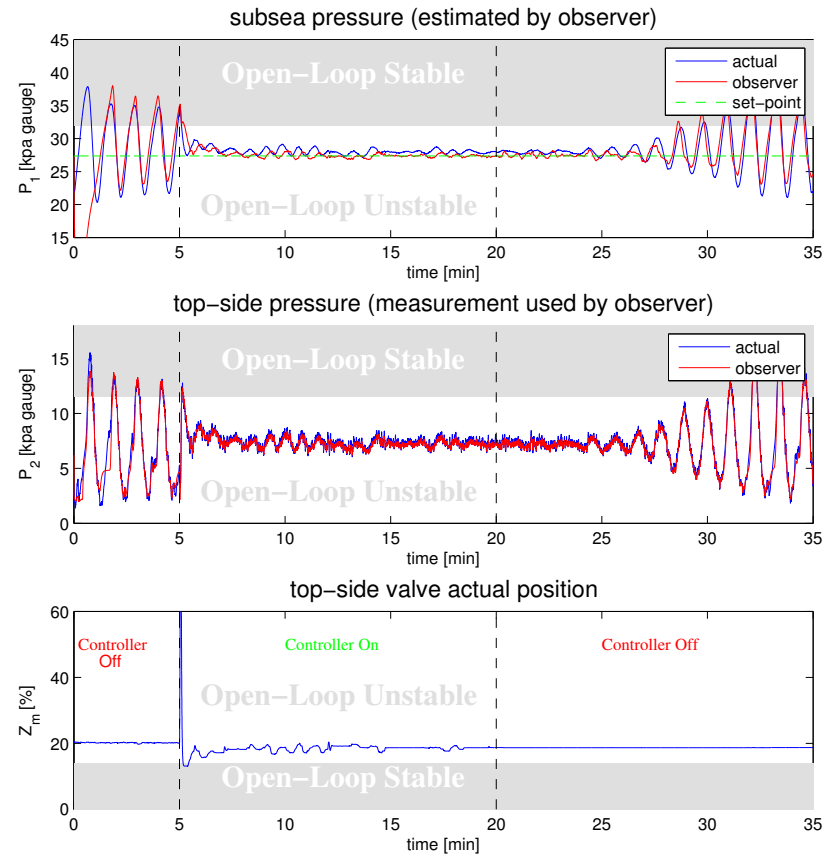

Fig. 4. Control using High-Gain observer and measuring top pressure $\left(P_{2}\right)$ with $20 \%$ valve opening

when the system can be stabilized. We do not present the result here due to space limitation. As shown in Fig. 2, the open-loop system switches from stable to slugging flow for valve openings $Z>15 \%$. We were able to stabilize the system up to $Z=20 \%$ by using nonlinear observers. However, as the valve opening increases, control becomes more difficult [14].

\section{B. Measuring subsea pressure}

Based on a controllability analysis in [3][14], it is much easier to stabilize the system by using the subsea pressure compared to the top-side pressure. The system could be stabilized up to $40 \%$ valve opening by controlling the subsea pressure with a simple PI controller (Fig. 6).

Next, we considered state feedback control based on observers using the subsea pressure measurement. We can construct observers for the subsea pressure measurement, as done in the previous section, by transforming the first state by choosing $z_{1}=P_{1}$ for the Luenberger observer and the fast UKF. However, by using a fast nonlinear observer (highgain Luenberger and fast UKF) with the subsea pressure measurement, it was not possible to stabilize the system.

Fig. 7 shows the result of estimation by a Luenberger observer with a large gain $\left(\epsilon=10^{-4}\right)$ in open-loop, where the subsea pressure is the measurement used by the observer. The top-side pressure and its related state variables are not correctly estimated, and as a consequence, using them in state-feedback cannot stabilize the system. This happens when we increase gain of the observer for robustness of the closed-loop system.

We used a linear Kalman Filter with the subsea pressure measurement, and it was possible to stabilize the system
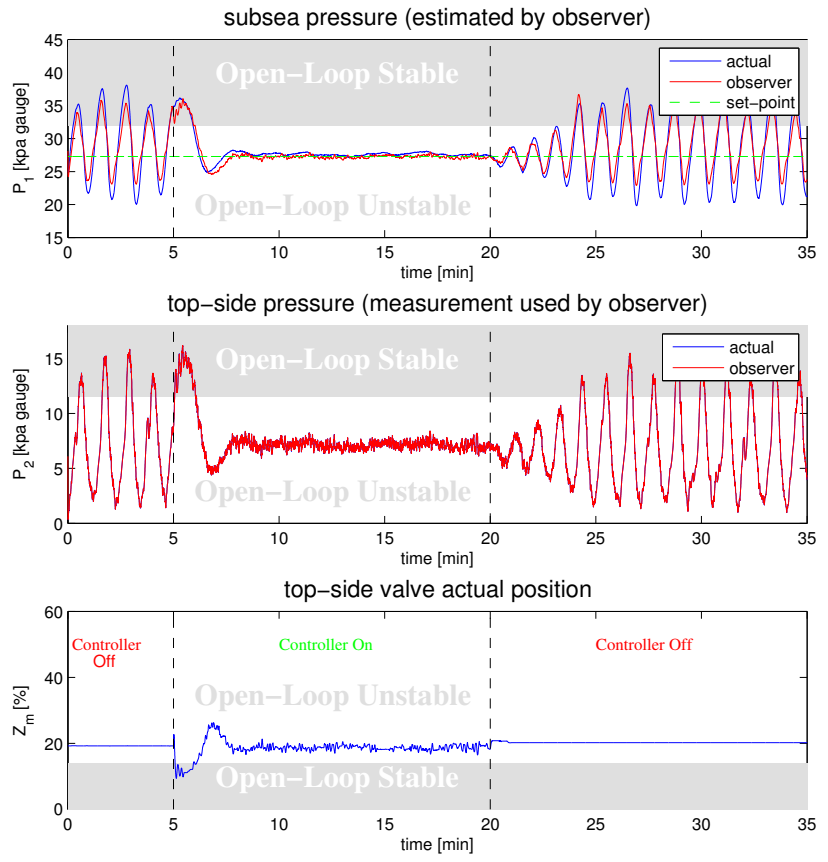

Fig. 5. Control using Fast UKF and measuring top pressure $\left(P_{2}\right)$ with $20 \%$ valve opening

up to $Z=40 \%$ valve opening. As shown in Fig. 8, an aggressive control action is needed to stabilize the system at this operating point.

Since the nonlinear observers around the nominal operating point have the same behavior as a linear observer, we started the experiment in closed-loop to keep the system always around its equilibrium point. Using this start-up approach, among the three nonlinear observers, only the Fast UKF was able to stabilize the system. However, it was not robust against disturbance in boundary (inflow) conditions. We summarize performance of different observers for state estimation in Table I, and performance of different methods for stabilizing control in Table II.

TABLE I

STATE ESTIMATION USING DIFFERENT OBSERVERS

\begin{tabular}{|c|c|c|}
\hline method \measurement & subsea pressure & top pressure \\
\hline fast linear observer & working & not working \\
fast nonlinear observer & not working & working \\
slow nonlinear observer & working & working \\
\hline
\end{tabular}

TABLE II

STABILIZING CONTROL USING DIFFERENT METHODS

\begin{tabular}{|c|c|c|}
\hline method \measurement & subsea pressure & top pressure \\
\hline $\begin{array}{c}\text { linear controller }\left(\mathrm{PI}, \mathcal{H}_{\infty}\right) \\
\text { fast linear observer } \\
\text { fast nonlinear observer } \\
\text { slow nonlinear observer }\end{array}$ & $\begin{array}{c}\text { working } \\
\text { working } \\
\text { not working } \\
\text { not robust }\end{array}$ & $\begin{array}{c}\text { not working } \\
\text { not working } \\
\text { working } \\
\text { not robust }\end{array}$ \\
\hline max. valve opening & $40 \%$ & $20 \%$ \\
\hline
\end{tabular}




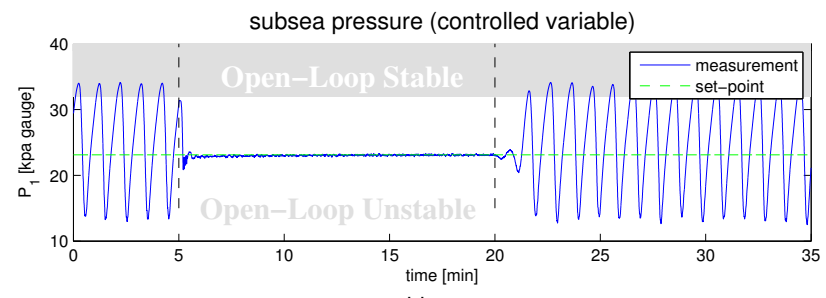

top-side pressure
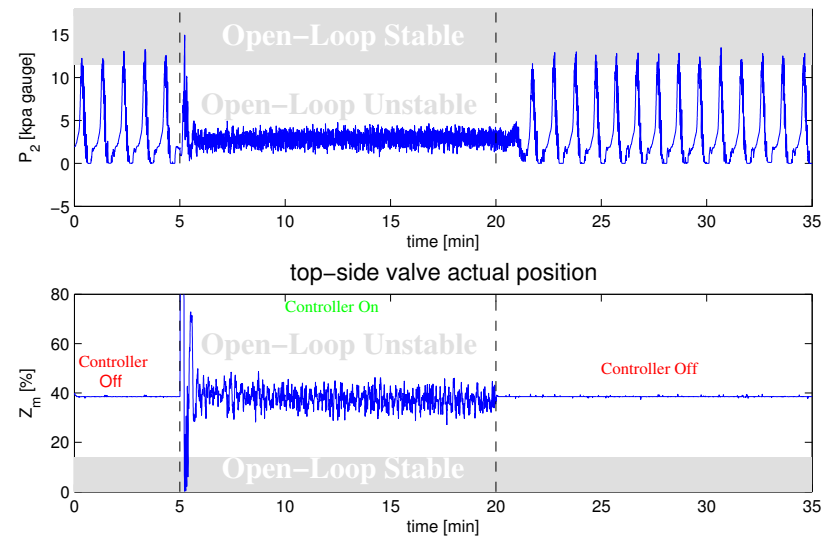

Fig. 6. Control using PI controller $\left(K_{c}=-10, T_{i}=200 \mathrm{sec}\right)$ and measuring subsea pressure $\left(P_{1}\right)$ with $40 \%$ valve opening
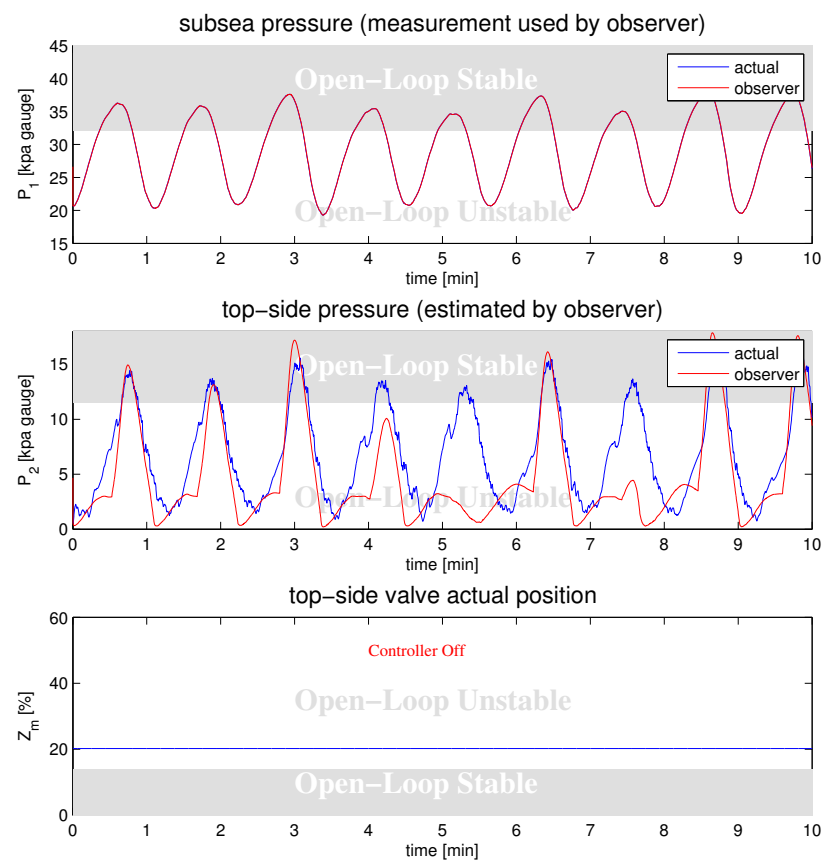

Fig. 7. Open-loop estimation using High-Gain observer and measuring subsea pressure $\left(P_{1}\right)$ with $20 \%$ valve opening
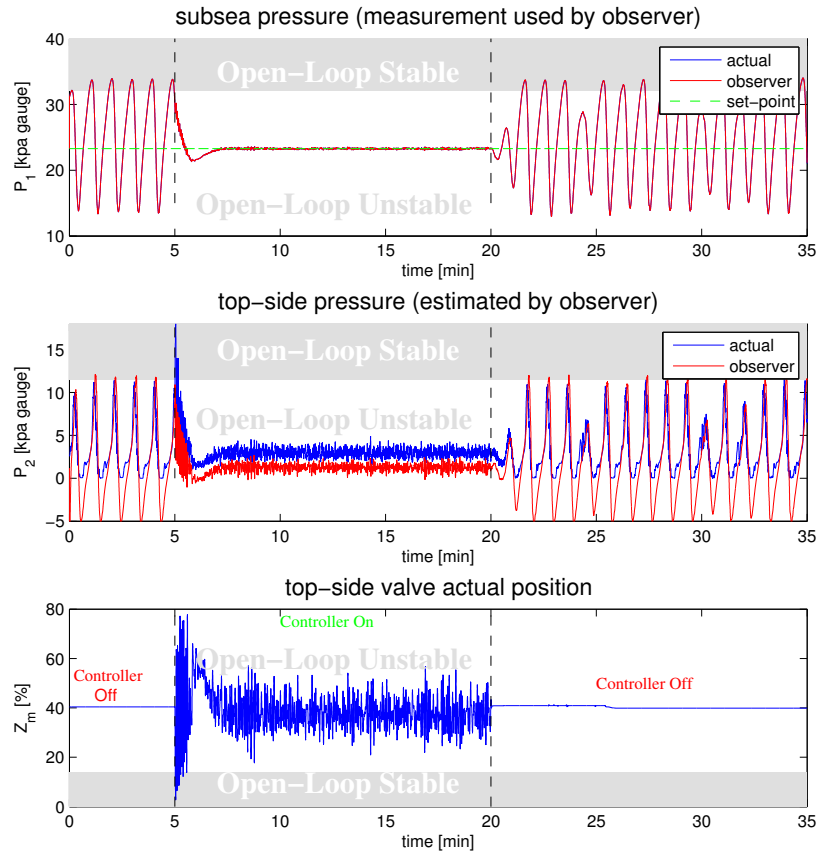

Fig. 8. Control using linear $\mathrm{KF}$ and measuring subsea pressure $\left(P_{1}\right)$ with $40 \%$ valve opening

\section{DISCUSSION}

\section{A. Measuring top-side pressure}

We tried to control the top-side pressure directly using a PI controller. Different tunings were used without any success. However, if we use the top-side pressure measurement to estimate the states by using a fast nonlinear observer, the system can be stabilized by using estimated states in the linear state-feedback controller. We make two hypotheses to explain this observation:

- The top-side pressure dynamics is highly nonlinear towards the valve

- The RHP-zero (inverse response) in top pressure dynamics limits the controllability

One or both of these two reasons can be correct. We tried to stabilize the system by using a fast linear observer, but it was not possible. This confirms that the first factor (nonlinearity effect) is important. The nonlinear observer counteracts the nonlinearity of the dynamics and the states are more linear towards the valve.

The RHP-zero dynamics leads to high peaks of the sensitivity transfer functions and they impose a limitation on the controllability of the system. The lowest achievable peaks of sensitivity and complementary sensitivity transfer functions, denoted $M_{S, \text { min }}$ and $M_{T, \text { min }}$, are closely related to the distance between the unstable poles $\left(p_{i}\right)$ and zeros $\left(z_{i}\right)$. Considering SISO systems, for any unstable (RHP) zero $z$ :

$$
\|S\|_{\infty} \geq M_{S, \min }=\prod_{i=1}^{N_{p}} \frac{\left|z+p_{i}\right|}{\left|z-p_{i}\right|} .
$$


This bound for our system at the operating point $Z=20 \%$ with the top-side pressure as the output is around 2, which seams not to be very limiting. However, this bound does not consider how much bandwidth is needed to stabilize the unstable system. We investigated this fact more closely by using a $\mathcal{H}_{\infty}$ controller for control of the top-side pressure. We need a fast control action, with about $1 \mathrm{sec}$ response time, to stabilize the system. When applying a bandwidth of $1 \mathrm{rad} / \mathrm{sec}$ in loop shaping specifications of the $\mathcal{H}_{\infty}$ controller, the peak of the sensitivity transfer function increases to 4 . This is called the water-bed effect. If we push down the sensitivity transfer function in low frequencies, it will increase in other frequencies [15].

Since the standard UKF (a nonlinear, but slow observer) was not robust in the closed-loop system, we realize that the time-scale separation requires a fast observer and it has to be faster than the RHP-zero dynamics of the top-pressure.

\section{B. Measuring subsea pressure}

Slow nonlinear observers can produce estimates of the topside pressure and its related states when measuring the subsea pressure. However, when increasing the observer gain they fail. In contrary, the fast nonlinear observers work well when measuring the top-side pressure.

The structure of the model for the high-gain observer in [11] is introduced as a chain of integrators and the measured state is at the end of the chain (e.g. measuring the position and estimating velocity in mechanical systems). The highgain observer behaves like a differentiator and it gives a more accurate estimate by using higher gain, similar to calculating derivative by finite difference by using smaller step.

If we consider the structure of the four-state model [10], we can find a chain of integrators. The mass of gas in the riser, which is closely related to the top-pressure, is the integral of the gas flow rate at the riser-base, which is determined by the subsea pressure. Roughly speaking, the subsea pressure is related to derivative of the top-side pressure, and as a result, fast observer works very well by measuring the top pressure.

On the other hand, by measuring the the subsea pressure, we actually need to integrate it to find the top-side pressure and the associated state variables. There are two problems arising in estimation by integrating. First, for a good estimate we need a larger integration time; this is the reason for success of slow observers in estimation. The second problem is that it is very sensitive to modeling errors and disturbances. When an unknown model change or disturbance happens, the observer continues to integrate the wrong conditions, and finally it will fail as shown in Fig. 7. As mentioned above, even when we start the system in closed-loop to keep it always near the stationary point, it was not robust against disturbances in inflow conditions.

In the case of the linear observer, we assume the correct stationary point and we only deal with the deviations. Consequently, as shown in Fig. 8, the estimation of the linear observer is less accurate, but it does not fail to stabilize the system.

\section{CONCLUSION}

The four-state simplified model shows a good agreement with the experiments. Anti-slug control using top-side pressure is possible by use of fast nonlinear observers, but the operating range of this scheme is less than using the subsea pressure and PI controller. We could stabilize the system by using the top-side pressure up to $20 \%$ valve opening, while by controlling the subsea pressure using a PI controller we could open the valve up to $40 \%$. The larger valve opening means higher rate of oil production. Surprisingly, the fast nonlinear observers failed to stabilize the system by using the subsea pressure.

Moreover, we found that both nonlinearities and the RHPzero dynamics are important problematic factors in control of the top-side pressure. Fast nonlinear observers can make the control possible in a limited range, but they can not bypass fundamental limitations of the system completely.

Having the subsea pressure available, a linear observer and even a simple PI controller are performing better than the nonlinear observers.

\section{REFERENCES}

[1] J.-M. Godhavn, M. P. Fard, and P. H. Fuchs, "New slug control strategies, tuning rules and experimental results," Journal of Process Control, vol. 15, p. 547557, 2005.

[2] H. Sivertsen, V. Alstad, and S. Skogestad, "Medium-scale experiments on stabilizing riser-slug flow," SPE Projects, Facilities \& Construction, vol. 4, no. 4, pp. 156-170, SPE no. 120040, 2009.

[3] E. Storkaas and S. Skogestad, "Controllability analysis of two-phase pipeline-riser systems at riser slugging conditions," Control Engineering Practice, vol. 15, no. 5, pp. 567-581, 2007.

[4] G. O. Eikrem, L. Imsland, and B. Foss, "Stabilization of gas lifted wells based on state estimation," in IFAC International Symposium on Advanced Control of Chemical Processes, Hong Kong, China, 2004.

[5] O. M. Aamo, G. O. Eikrem, H. Siahaan, and B. Foss, "Observer design for gas lifted oil wells," in American Control Conference. IEEE, 2004.

[6] G. O. Eikrem, O. M. Aamo, H. Siahaan, and B. Foss, "Anti-slug control of gas-lift wells - experimental results," in 6th IFAC Symposium on Nonlinear Control Systems, Stuttgart, Germany, 2004.

[7] F. Di Meglio, G.-O. Kaasa, N. Petit, and V. Alstad, "Reproducing slugging oscillations of a real oil well," in 49th IEEE Conference on Decision and Control, Atlanta, Georgia, USA, 2010.

[8] F. Di Meglio, "Production de pétrole: étude dynamique et contrôle des écoulements à bouchons," Phd. Thesis, l'Ecole des mines de paris, 2011.

[9] F. Di Meglio, G.-O. Kaasa, and N. Petit, "A first principle model for multiphase slugging flow in vertical risers," in Joint 48th IEEE Conference on Decision and Control and 28th Chinese Control Conference, Shanghai, China, 2009.

[10] E. Jahanshahi and S. Skogestad, "Simplified dynamical models for control of severe slugging in multiphase risers," in 18th IFAC World Congress, Milan, Italy, Auguest 2011, pp. 1634-1639.

[11] H. K. Khalil, Nonlinear Systems, Third Edition. Upper Saddle River, New Jersey: Prentice Hall, 2002.

[12] S. J. Julier and J. K. Uhlmann, "A new extension of the kalman filter to non-linear systems," in AeroSense: The 11th Int. Symp. A.D.S.S.C., 1997.

[13] N. Boizot, E. Busvelle, and J.-P. Gauthier, "An adaptive high-gain observer for nonlinear systems," Automatica, vol. 46, no. 9, pp. 1483$1488,2010$.

[14] E. Jahanshahi, S. Skogestad, and A. H. Helgesen, "Controllability analysis of severe slugging in well-pipeline-riser systems," in IFAC Workshop - Automatic Control in Offshore Oil and Gas Production, Trondheim, Norway, May 2012.

[15] S. Skogestad and I. Postlethwaite, Multivariable Feedback Control: Analysis and Design. Chichester, West Sussex, UK: Wiley \& Sons, 2005. 